\title{
The Severity of Dependence Scale had high sensitivity and specificity for detecting benzodiazepine dependence
}

de las Cuevas C, Sanz EJ, de la Fuente JA, et al. The Severity of Dependence Scale (SDS) as screening test for benzodiazepine dependence: SDS validation study. Addiction $2000 \mathrm{Feb}$;95:245-50.

QUESTION: Does the Severity of Dependence Scale (SDS) accurately detect benzodiazepine dependence among people who use benzodiazepines regularly?

\section{Design}

A blinded comparison of the Severity of Dependence Scale with the Composite International Diagnostic Interview (CIDI).

\section{Setting}

A mental health outpatient service in the Canary Islands.

\section{Participants}

100 participants who were 18-75 years of age $(75 \%$ women), had a neurotic diagnosis (ICD-10 F4 category), continuously used benzodiazepines daily for $\geqslant 3$ months (mean $22 \mathrm{mo}$ ), and had a stable maintenance dose of their benzodiazepine equivalent to $5-50 \mathrm{mg}$ /day of diazepam. Exclusion criteria were current diagnosis of schizophrenia or organic brain syndrome, recent history of other substance abuse (within previous $12 \mathrm{mo}$ ), presence of any acute or unstable medical or psychiatric problem, or any problem that may affect understanding of the test.

Description of test and diagnostic standard The wording of the original 5 SDS items was adapted to cover benzodiazepines as drugs used. All participants completed the SDS when they had stable symptoms or were symptom free. Each item was scored by the participant on a 4 point scale; high scores reflected greater severity. The diagnostic standard was the CIDI, which was given by one psychiatrist who was blind to SDS results. A current diagnosis of benzodiazepine dependence was given if $\geqslant 3$ symptoms occurred in the previous month.

\section{Main outcome measures}

Sensitivity, specificity, positive and negative likelihood ratios, and area under the receiver operating characteristic (ROC) curve.

\section{Main results}

$48 \%$ of participants were diagnosed as having current benzodiazepine dependence on the CIDI. The table shows the sensitivity, specificity, and likelihood ratios for a cut off score of 7 on the SDS. The area under the ROC curve was 0.99 , which means that the SDS can correctly detect patients who have benzodiazepine dependence in $99 \%$ of cases.

\section{Conclusion}

The Severity of Dependence Scale had high sensitivity and specificity for detecting benzodiazepine dependence.
Test characteristics of the Severity of Dependence Scale for detecting current benzodiazepine dependence using a cut off score of 7 *

\begin{tabular}{|c|c|c|c|}
\hline $\begin{array}{l}\text { Sensitivity } \\
(95 \% \mathrm{CI})\end{array}$ & $\begin{array}{l}\text { Specificity } \\
\text { (CI) }\end{array}$ & +LR (CI) & $-\mathrm{LR}(\mathrm{Cl})$ \\
\hline $\begin{array}{l}98 \% \\
(89 \text { to } 100)\end{array}$ & $\begin{array}{l}94 \% \\
\text { (84 to } 99 \text { ) }\end{array}$ & $\begin{array}{l}17.0 \\
(6.3 \text { to } 49.4)\end{array}$ & $\begin{array}{l}0.02 \\
(0.00 \text { to } 0.12)\end{array}$ \\
\hline
\end{tabular}

+LR=likelihood ratio for a positive test; -LR=likelihood ratio for a negative test. Diagnostic terms defined in glossary; LRs and $\mathrm{Cl}$ calculated from data in article.

\section{COMMENTARY}

The study by de las Cuevas et al examines the potential of an easy to understand, 5 question, self report screening test (the SDS) to identify benzodiazepine dependence, and found it to be remarkably successful. This scale, which takes only 1-3 minutes to administer, correctly classified $92 \%$ of those who were benzodiazepine dependent by using a cut off score of 7 or above. Only $6 \%$ were classified as dependent when they were not, and $2 \%$ as not dependent when they were, compared with the gold standard diagnosis of benzodiazepine dependence, which was made using a complex research interview (the CIDI) that elicits the criteria specified in $I C D-10$ and $D S M-I V$.

Interestingly, patients' views on whether they were dependent or not correlated poorly with the presence of actual dependence. Indeed, $38 \%$ of those who thought they were dependent were not, and $12 \%$ of those who were not dependent thought they were. Sex made no difference to the prevalence rates in this outpatient setting.

Thus, the SDS appears to be a useful screening test in outpatients on a stable long term dose of medium or long half life benzodiazepines. Whether it would be so useful in short term use $(<3 \mathrm{mo})$ or with short half life hypnotic benzodiazepines is uncertain. Although it has not been proved that the SDS can predict success at reduction or detoxification, this may well be the case because it predicts dependence. The same scale has also been shown to be useful for determining dependence on other drugs, including opiates and amphetamines. ${ }^{1}$

Dependence on benzodiazepines is an important clinical problem occurring in $15-44 \%$ of those prescribed benzodiazepines long term. The clinical usefulness of this scale is threefold: firstly, busy clinicians and those without experience in assessing benzodiazepine dependence will be able to use it; secondly, it gives a measure of the severity of dependence which can change over time; and thirdly, it allows the rapid identification of patients with probable benzodiazepine dependence on whom resources may need to be targeted during attempts to cut down or achieve abstinence. Fergus D Law, BSc, MBChB, MRCPsych University of Bristol Bristol, UK

1 Gossop M, Darke S, Griffiths P, et al. The Severity of Dependence Scale (SDS): psychometric properties of the SDS in English and Australian samples of heroin, cocaine and amphetamine users. Addiction 1995;90:607-14.
Source of funding FUNCIS (Fundación Canaria de

Investigación y Salud) of the Canary Islands Government.

For correspondence: Professor $C$ de las Cuevas Castresana, Mental Health Service, Canary Islands Health Service, C/ Pérez de Rozas, 5-3ㄱ, 38004 Santa Cruz de Tenerife, Spain.Fax +34922 475735 